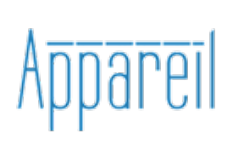

Appareil

$15 \mid 2015$

L'art dans le tout numérique

\title{
L'appareil photographique : entre prise de vue et prise de pouvoir
}

Natalia Calderón

\section{(2) OpenEdition}

1 Journals

Édition électronique

URL : http://journals.openedition.org/appareil/1375

DOI : 10.4000/appareil. 1375

ISSN : 2101-0714

Éditeur

MSH Paris Nord

Référence électronique

Natalia Calderón, «L'appareil photographique : entre prise de vue et prise de pouvoir », Appareil [En

ligne], 15 | 2015, mis en ligne le 10 janvier 2012, consulté le 02 août 2020. URL : http://

journals.openedition.org/appareil/1375; DOI : https://doi.org/10.4000/appareil.1375

Ce document a été généré automatiquement le 2 août 2020.

\section{(c) (i) ()}

Appareil est mis à disposition selon les termes de la Licence Creative Commons Attribution - Pas d'Utilisation Commerciale - Pas de Modification 4.0 International. 


\title{
L'appareil photographique : entre prise de vue et prise de pouvoir
}

\author{
Natalia Calderón
}

\section{Photographie et prise de terre}

1 Ronald Kay, dans son texte L'espace d'ici' ${ }^{1}$, détermine d'une façon très précise le moment où l'appareil photographique s'installe en Amérique latine :

Ces prises (photographiques) sont des signaux optiques des points géographiques découverts. Elles constituent des morceaux des preuves de leur réelle (et pas fantastique) existence; elles sont la nouvelle documentée de leur conquête. À la fois, elles connotent l'inventaire de ce qu'il reste à dominer, à occuper, à exploiter. Elles sont d'une certaine façon des cibles. Graphiquement, la prise photographique dans le Nouveau Monde est une prise de possession. ${ }^{2}$

2 Les prises de vue ne se présentent donc pas comme de simples images. Il s'agit plutôt d'un moyen de réaliser la cartographie d'un territoire qui permettra son occupation, avec des données plus précises apportées par l'appareil photographique. La question qui se pose est la suivante : est-ce que la prise de vue en Amérique latine doit toujours être considérée comme une prise de pouvoir? La réponse semble être affirmative, au moins à ses débuts.

3 On peut dire qu'il existe une certaine contemporanéité entre l'arrivée de l'appareil photographique en Amérique latine et la formation de ses premières nations. Les nations sont apparues avec les mouvements indépendantistes qui ont mis fin à la domination espagnole. Les nations, qui se créeront sous le signe de la liberté, à l'instar de la Révolution française, perpétueront paradoxalement leur domination sur les peuples indigènes, domination entreprise lors de la conquête. L'appareil photographique se présente alors comme le principal moyen avec lequel les nations vont entreprendre l'occupation des territoires des peuples autochtones. 
4 Tout ordre spatial en Amérique latine est fondé sur son caractère supposé de « Nouveau Monde». Cette dénomination sous-entend l'idée d'un territoire vide, où les lignes directrices n'ont pas encore été déterminées.

Il s'agit d'un espace qui, du fait qu'il est nouveau, n'est pas soumis à une loi qui oriente sa propre détermination. C'est la nouveauté de l'Amérique latine qui la caractérise comme un territoire indéterminé. Pourtant, comme le signale Carl Schmitt, ${ }^{3}$ en présence d'un territoire «libre », comme cela a été le cas de l'Amérique latine, l'être européen s'attribue le droit de déterminer son propre ordre et celui de l'étranger. L'Europe pourra donc effectuer son droit à la détermination de ce territoire. Ce droit de détermination européen est fondé par un ordre européocentrique.

De ce fait, la conquête espagnole s'érige comme l'événement fondateur de toute légitimité ultérieure en Amérique latine. Elle est à la fois une prise de territoire et une prise de pouvoir.

7 L'événement fondateur de cette prise, Schmitt l'appelle nomos, dont le sens étymologique est le suivant :

Le mot grec pour la première mensuration qui fonde toutes les mesures ultérieures, pour la première prise de terre en tant que première partition et division de l'espace, pour la partition et la répartition originelles, c'est : nomos. ${ }^{4}$

Il s'agit d'une définition qui montre comment cet événement s'installe comme une localisation spatiale, et en même temps, comme l'établissement d'un certain ordre à partir duquel toutes les autres normes doivent s'établir.

9 La principale conséquence qui découle de l'événement de la conquête espagnole en Amérique latine est l'annulation de tout pouvoir d'autodétermination.

10 Schmitt signale que le nomos instauré pendant la conquête se donne en vertu du droit des gens qui permet de séparer l'ordre et le chaos. L'Amérique latine appartenait au chaos, la normalisation s'impose donc à partir du nomos. Il s'agit d'une logique exclusive qui ne reconnaît pas l'autre comme légitime. D'après Schmitt, c'est à partir du $\mathrm{XVIII}^{\mathrm{e}}$ et $\mathrm{XIX}^{\mathrm{e}}$ siècle que cette situation change, avec l'instauration d'États qui se construisent sous la reconnaissance réciproque de leur légitimité.

11 En Amérique latine les mouvements indépendantistes marquent le début du processus de construction des premières nations. Elles en ont fini avec le nomos déterminé de l'extérieur pour commencer le développement d'un nouveau nomos, cette fois autogéré. Il s'agit du commencement zéro de la loi. Mais cette nouvelle possibilité d'autodétermination des nations en Amérique latine aura aussi généré une nouvelle forme d'exclusion et de non-reconnaissance. Il s'agit de l'exclusion des peuples autochtones qui appartiendront plutôt au chaos qu'au nomos, dit national. Il s'agit d'un paradoxe, qui est toujours d'actualité, l'impossibilité d'instaurer un seul nomos au sein d'un territoire national, sans avoir comme conséquence un régime d'exclusion.

De quelle façon se dessine la détermination de l'espace en Amérique latine au XIX siècle, époque dans laquelle les nations sont en train de développer leurs propres nomos après la libération de celui imposé par l'Espagne et plus généralement par l'Europe ? On peut dire que c'est à travers la photographie que cette topo-nomologie, comme dit Derrida $^{5}$, va se déployer.

13 Quel sera alors le caractère spécifique qui rend l'appareil photographique capable de développer cet événement fondateur? Il faut s'interroger tout d'abord sur le caractère 
technique spécifique de l'appareil photographique comme surface privilégiée d'inscription d'un tel nomos.

14 La photographie s'est installée en Amérique latine comme photographie analogique, c'est-à-dire une structure technique inouïe que l'on va nommer « photographies du ça a été ». En prenant la terminologie de Ronald Barthes, la photographie a comme résultat la découverte de la sensibilité de la lumière à certaines substances chimiques, qui ont permis de capter et d'imprimer l'écriture de la lumière elle-même.

Barthes considère que la photographie est une émanation du réfèrent par le fait qu'elle est l'écriture de la lumière, qu'elle est le spectrum de l'objet imprimé sur une surface. Barthes voit en la photographie la preuve de la matérialité des objets, de leur existence toujours passée mais qui malgré tout adhère.

C'est cette idée de la valeur d'objectivité de la photographie qui aura été le fondement de son usage comme document, comme archive. Barthes signale :

Peut-être avons-nous une résistance invisible à croire au passé, à l'histoire, sinon sur la forme de mythe. La photographie pour la première fois, fait cesser cette résistance : le passé est désormais aussi sûr que le présent. ${ }^{6}$

17 Si le passé pouvait auparavant retrouver la réalité et la certitude que seul le présent possède, désormais on est en condition de dire que la photographie sera la surface sur laquelle le passé s'inscrit de manière objective, inaugurant la possibilité réelle d'une historiographie.

18 C'est de cette façon qu'on peut penser la photographie comme des images faites pour être consultées comme un répertoire des images du monde, archivées, classifiées. Cela permettra de les comparer, de les analyser pour, à partir de là, développer une recherche d'ordre historique-scientifique.

19 Le caractère de preuve du réel aura alors permis à la photographie de se transformer en archive, c'est-à-dire, en une origine à partir de laquelle sera possible la construction d'une topo-nomologie. Lieu où s'inscrit une loi, tel que le comprend Derrida dans Mal d'archive:

Avec un tel statut, les documents, qui ne sont pas toujours des écritures discursives ne sont gardés et classés au titre de l'archive qu'en vertu d'une topologie privilégiée. Ils habitent ce lieu particulier, ce lieu d'élection où la loi et la singularité se croisent dans le privilège. ${ }^{7}$

Chez Derrida, l'archive c'est à la fois un lieu et une loi, c'est une matérialité singulière qui inaugure une loi à partir de laquelle toute autre normativité pourra s'inscrire, de la même façon que Schmitt comprend le nomos originaire. Il est important de signaler que, pour Derrida, l'archive ne se limite pas à son caractère discursif; cette idée nous permet de considérer les images photographiques prises en Amérique latine pour les gouvernements comme une forme d'archive.

21 C'est de cette façon que les nations latino-américaines se sont appropriées la photographie en tant que possibilité de création d'une archive de leurs propres imaginaires nationaux. Archive première peut-on dire, qui inaugure la détermination spécifique de chaque nation. Comme on l'a déjà dit, les mouvements indépendantistes auront recours à l'idée du commencement zéro, donc cette reconstruction nouvelle aura besoin d'une surface sur laquelle inscrire ce nouveau début.

Le plus intéressant sera alors de montrer comment, malgré la considération du statut ontologique de la photographie, elle doit être considérée comme un appareil, en ce sens 
que «Les appareils doivent extraire leur matériau non du réel sensible mais du plasma imaginal. $»^{8}$ La photographie en tant que prise de pouvoir montre clairement comment l'appareil aura été capable d'être non seulement une preuve ontologique, mais aussi de produire du sens lui-même. ${ }^{9}$

Il ne faut pas perdre de vue que si la photographie se présente bien comme un aidemémoire, comme une prothèse qui archive le passé, cette archive est malgré tout une répétition. Toute archive garde ce qu'on pense qu'il est important de garder. Il s'agit d'une hiérarchie des événements, qui sont gardés selon un certain ordre. Chez Derrida, l'archive n'est pas seulement le lieu de stockage d'un passé indépendant de son archivage, il s'agit plutôt de penser l'archive comme ce qui va structurer le passé luimême. Dans ce sens, Barthes considère encore le passé comme indépendant de la façon de l'archiver.

Tout au contraire, il faut penser la photographie comme archive, comme une façon de structurer le passé qui assure sa reproduction. Le passé revient par le moyen de l'appareil photographique, mais c'est un passé photographique. Il est donc nécessaire de penser les imaginaires nationaux en Amérique latine, comme des inscriptions du passé sous la structure donnée par la photographie.

Après les mouvements indépendantistes s'est installée une nouvelle cartographie en Amérique latine, suite à la nécessité de déterminer plus précisément les limites de chaque nation.

L'idée de nation liée à l'idée de territoire, à la délimitation de frontières nationales, sera fondamentale pour pouvoir comprendre l'importance de la photographie à ce momentlà.

Quand on comprend la légitimité de la photographie comme créatrice d'un espace, d'un territoire qui va former l'imaginaire national, on comprend les efforts des gouvernements latino-américains pour envoyer des expéditions photographiques capables de créer un inventaire précis du territoire national. On peut parler dans ce sens-là d'une carto-photographie ou de nomo-photographie, d'une archive qui aura comme prétention la délimitation d'un territoire, la création d'un topos national pour, à partir de là, créer des instances de légitimation ou d'exclusion.

Dans ce sens là, il ne faut pas perdre de vue que la définition de nomos chez Schmitt a une connotation spatiale. Il s'agit du marquage de la terre qui permettra le développement d'autres considérations ou de lois plus abstraites.

La prise de terre et son marquage sont des événements historiques fondateurs d'ordre spatial. La photographie peut-être considérée comme un appareil qui met en place ce marquage, cette prise de terre.

D'un point de vue symbolique, pourtant bien concret, la photographie est l'élément fondateur qui a permis la prise de terres. Tout d'abord parce que l'arrivée de la photographie en Amérique latine au XIX siècle est contemporaine de cette nouvelle prise de terre faite par les gouvernements des premières nations latino-américaines. Cette démarche permettra d'en finir avec l'ancien nomos déterminé par la conquête espagnole-portugaise. Cette nouvelle prise de terre se déploiera avec l'appareil photographique selon un nouveau mode de structuration temporelle. La photographie est donc déterminante pour toute investigation qui cherche à déterminer le nomos originaire qui commence à se déployer au XIX ${ }^{e}$ siècle. 
31 Nous allons parler de trois albums photographiques qui apparaissent comme exemplaires au titre du rapport entre la prise de vue et la prise de pouvoir en Amérique latine.

Le premier s'appelle «El album del Santa Lucía » (L'album de Sainte Lucie) ${ }^{10}$, édité en 1874 et dirigé par le président du Chili de l'époque, Benjamin Vicuña Mackenna.

Le deuxième album s'appelle « Album de vistas. Expedición al río Negro. Abril a Julio de $1879 »$ (Album de vues. Expédition à la rivière Noire. Avril à juin 1879) de Antonio Pozzo. ${ }^{11}$

Le dernier, qui concerne la même expédition, est l'« Album de Encina, Moreno y cía. $1883 »^{12}$

\section{L'album de la butte Santa Lucía}

32 L'album de la butte Santa Lucía se présente comme le paradigme de l'instauration d'un nomos originaire, car depuis l'époque de la conquête elle a été le lieu par excellence d'inscription d'un nouvel ordre. Schmitt signale :

Tant que les hommes et les peuples ont encore un avenir et pas seulement un passé, un nouveau nomos naîtra dans les formes toujours nouvelles que prendra le cours de l'Histoire. ${ }^{13}$

L'album du Santa Lucía marque le changement de l'ancien nomos de l'époque de la conquête vers le nouveau nomos de l'époque de la Nation.

La butte Santa Lucía est située au centre de la ville de Santiago, la capitale du Chili. Historiquement, elle était un lieu sacré pour les indigènes, un lieu du culte, symbole du pouvoir inamovible de la nature, lieu où habitent les âmes des morts. La butte était également considérée comme un lien entre les trois mondes : le Ciel, la Terre et l'Océan (qui sont censés être liés par des connexions souterraines). Cette butte a été nommée paradoxalement par les Indiens « Huelén », c'est-à-dire, « la douleur ».

Quand Pedro de Valdivia arrive à Santiago, il monte sur la butte Huelén pour faire la première prise de terre, pour établir la première mensuration de la ville, c'est-à-dire pour fonder un nomos originaire. L'histoire signale que Valdivia a tracé le plan de la ville sur la terre même de la butte Huelén. Il s'agit donc d'une prise de pouvoir faite dans une localisation spécifique qui rendra possible tout ordre postérieur.

Ce marquage sur la terre a impliqué aussi une renomination, car le nom indigène Huelén est effacé et dorénavant la butte s'appellera Santa Lucía. Dans cet événement où s'établit le nomos, toute une tradition précolombienne sera effacée, étant considérée comme un élément vide de toute signification, sans détermination propre, en conséquence appropriable. ${ }^{14}$

37 On peut dire qu'avec le mouvement indépendantiste, qui a donné naissance aux différentes nations de l'Amérique latine, on met fin à l'établissement d'un nomos européocentriste. Pourtant, le nouveau nomos déterminé par les nations latinoaméricaines n'aura pas fini avec un régime d'exclusion à l'intérieur des nations ellesmêmes.

C'est Benjamin Vicuña Mackenna qui mènera les transformations de la butte Santa Lucía au XIX ${ }^{e}$ siècle. La butte Santa Lucía sera de nouveau le lieu choisi pour cette nouvelle prise de terres, comme elle l'a été avant le lieu où Valdivia instituera le régime colonial. 

phrase suivante :

Ce double nomos (le colonial et le national) est inscrit plus concrètement sur un grand rocher qui porte une sculpture de la déesse de Caracas. "L'album du Santa Lucía » le montre clairement. Les deux faces du rocher signalent les deux différentes prises de terres, les deux différents nomos qui y ont été inscrits.

La façade sud du rocher signale «Huelén! 1541 » (fig. 1) date de la fondation de Santiago par Pedro de Valdivia. La façade nord indique «Promenade de Santa Lucía inaugurée solennellement le 17 septembre de 1872. Cuvre de dieux dont le peuple avec ses offrandes la fait sienne " (fig. 2). Deux faces d'un même rocher, double inscription de deux nomos différents.

Il est important de signaler que le président du Chili Benjamin Vicuña Mackenna, auteur de l'album du Santa Lucía, a écrit dans la première partie du texte de l'album la

la butte Santa Lucía, appelée Huelén (douleur) pour les primitifs et superstitieux habitants du Mapocho, est et a été une vraie merveille avant de donner le premier coup de houe qui l'a transformée. ${ }^{15}$

Cette citation montre clairement la manière dont on parle d'une nation divisée entre la civilisation proclamée par Vicuña Mackenna et la barbarie des peuples primitifs et superstitieux, habitant au sein d'une même nation.

L'album du Santa Lucía s'inscrit comme une lutte contre la barbarie vers l'idéal d'un monde moderne et civilisé.

L'album du Santa Lucía de Benjamin Vicuña Mackenna a comme ambition de montrer comment ces transformations se dérouleront, mais aussi comment l'appareil photographique est capable de structurer les transformations de l'espace pris.

À travers la description de la butte, Vicuña Mackenna nous propose la vision moderne qu'il essayera d'imposer.

Cet idéal moderne est incarné dans la butte Santa Lucía, il est issu de l'événement de la conquête, qui aura elle-même permis l'introduction de l'Amérique latine dans le monde civilisé et dans l'histoire universelle, au moment même où le développement urbain renforcera cette idée de progrès propre à la modernité.

Cette construction urbaine faite par Vicuña Mackenna possède une structuration qu'on peut nommer "photographique ». Il s'agit d'une nouvelle prise de terre comme on l'a déjà signalé, qui se structurera cette fois à travers l'appareil photographique.

Un des éléments les plus présents dans cette rénovation architecturale de la butte sera la reproduction. On peut parler d'un style architectural qui reprend différents styles de différentes époques. On peut trouver une copie du Cupidon du sculpteur français Bouchardon (œuvre exposée au musée du Louvre), des vases de style grec fabriqués dans une usine de Val d'Osne, une arche avec deux nymphes, un bouclier d'armes d'Espagne, une vierge en bronze et une petite place appelée Champs Élysées.

9 Parmi les statuesde la butte, on peut citer la statue d'un soldat de l'époque d'Attila et une autre d'un soldat saxon de la même époque. Vicuña Mackenna se réfère à ces statues et signale: "Les deux guerriers sont habillés avec des peaux d'animaux sauvages, comme les actuels Patagons [Indiens du sud du Chili], et ils se servent de beaux candélabres à gaz pour la promenade. $»^{16}$ 

d'une conception du style comme quelque chose qui a un rapport à l'histoire non linéaire. Meyer a déjà signalé que le style propre de la modernité, c'est l'éclectisme :

L'éclectisme qui caractérise l'art du xIXe siècle était bien plutôt une conséquence des conditions de vie dues au progrès intellectuel et matériel de toute notre civilisation. Ce progrès a fait tomber les frontières entre les pays; il nous rend accessibles, heure par heure, les fruits de l'arbre de toute l'humanité, il nous gratifie aussi d'un nouveau rapport au passé, aucune génération passée n'a jamais disposé de tels moyens pour enfoncer les portes millénaires ; aucune n'a jamais joui d'un panorama aussi complet, et chaque jour plus précis, de monuments artistiques de tous les temps et de tous les pays. Que le xIx siècle exploite ce merveilleux héritage du passé était presque une nécessité historique. ${ }^{17}$

On peut dire que l'appareil photographique a contribué à générer cet éclectisme, car il est déterminant dès lors de penser un nouveau rapport au passé fondé sur l'ubiquité, tandis qu'avant l'unicité était le fondement des événements et des œuvres d'art.

La conquête de l'ubiquité rend les œuvres d'art de tous les temps à la portée de tout le monde et n'importe où, et le rapport au temps se voit nettement modifié. Dans ce sens, la présence de différents styles exposés sur la butte Santa Lucía ne semble pas paradoxale. Il s'agit d'un accès au lointain, à travers la rupture de la différence existante entre l'ici et l'ailleurs.

D'ailleurs, le musée « historique-indigène " (fig. 3), comme l'appareil photographique, va contribuer au processus d'éclectisme. Créé à partir de "l'exposition coloniale " réalisée en 1873, le musée a été installé sur la butte Santa Lucía et propose une nouvelle manière d'aborder le passé. Il s'agit d'une collection d'œuvres de l'époque de la colonie espagnole, de quelques objets de la même époque et de quelques outils précolombiens.

Lessayera de récreer l'histoire passee d'un pays, en prenant des objets différents de provenances totalement différentes, pour les faire coexister dans un même espace. Le seul fondement de cette coexistence est le fait d'appartenir à une même époque. Mais est-ce qu'on peut dire qu'il existe un rapport réel entre le premier piano qui est arrivé au Chili et une prise de vue faite par l'ingénieur militaire Wood 40 ans avant la création du musée?

Quand on considère la problématique du musée, il faut réfléchir sur son caractère global qui donne le droit à toute possible mise en relation entre des objets complètement différents. Son caractère propre est qu'il fait abstraction des différentes provenances des objets qu'il rassemble. Le premier piano qui est arrivé au Chili doit avoir eu une utilité précise dans les salons qui à l'époque coloniale rassemblaient des classes aisées du pays. Maintenant dans le musée historique-indigène une telle utilité a été suspendue, il est exposé en tant que témoin d'une époque. Malraux signale : «les œuvres d'art ressuscitent dans notre monde de l'art, non dans le leur. $»^{18} C^{\prime}$ est de cette façon qu'on peut justifier le fait que toutes les œuvres pourront être rassemblées ; car leur destination spécifique a été perdue. Ce nouvel espace qui les rassemblera sera cette fois un espace global où les rapports entre œuvres totalement différentes sont possibles.

56 Est-ce qu'on peut penser que toute prise de pouvoir a besoin d'une inscription spécifique de tel événement fondateur? Est-ce qu'on peut dire que le musée est la surface sur laquelle une nation essayera d'inscrire son histoire? On peut dire que le 
musée va créer une continuité en oubliant les caractéristiques spécifiques de chaque œuvre et de chaque objet exposé.

Le nouveau nomos s'inscrira dans le musée et donnera cette fois une sorte de continuité avec le passé. Cette continuité sera possible par la suspension qui permet la réutilisation des objets du passé par des besoins actuels d'exposition.

Il s'agit de montrer comment cette nouvelle prise de pouvoir va s'intégrer à l'histoire générale d'une nation comme son aboutissement. Par contre, quand les œuvres ont leur spécificité propre, leur destination, une telle manipulation n'est pas possible; une œuvre faite pour le culte possède son fondement dans sa valeur cultuelle, quand on enlève cette destination cultuelle l'œuvre perd son fondement donc elle est perméable à une manipulation. La suspension de toute destination du monde colonial et précolombien implique la fin de son actualité et annonce une nouvelle époque.

Jean-Louis Déotte écrit que «Le musée est un supplément, une prothèse de ce qui n’a plus la force de se soutenir de soi-même, de ce qui ne va plus de soi, naturellement. ${ }^{19}$ Le passé colonial et précolombien se présente donc comme quelque chose qui a perdu son état naturel, et l'unique façon de retrouver ce rapport se fait à travers l'artifice. Mais il faut comprendre l'état naturel comme quelque chose qui est toujours appareillé, mais qui est appareillé avec des formes différentes qui, exposées dans un musée, montrent qu'elles ont perdu leur actualité. Le musée dans ce sens se présente comme un appareil capable de réactualiser ces œuvres qui ont perdu leur destination en les exposant, cette fois, comme des images ou comme des objets à contempler.

Il faut donc penser ce nouveau nomos de la butte Santa Lucía comme quelque chose qui s'inscrit par les moyens des appareils actuels. La photographie et le musée sont les surfaces privilégiées de cette inscription.

\section{L'album}

61 Ce n'est pas par hasard que Vicuña Mackenna a créé l'Album du Santa Lucía. Il signale dans la seconde partie du texte de l'album qu'il s'agit d'« une œuvre de propagande $»^{20}$.

Si la photographie va créer des images fragmentées du monde, l'album va fonctionner comme un mécanisme de restructuration de cette dispersion photographique. Il s'agit de deux modes différents de fonctionnement reliés dans l'album photographique. La photographie procède à un découpage du monde à travers les prises qui structurent différents points de vue. Par contre, l'album, comme l'archive, va fonctionner comme " des machines de dépôt, de collecte, de thésaurisation, qui accumulent et conservent. » ${ }^{21}$ L'album est une structure hybride faite à partir d'un découpage et remontage du monde.

Il faut dire que le remontage des prises de vue fait par l'album ne constitue pas le rétablissement d'un ordre préexistant, original. Tel serait un point de vue qui pense le réel comme une totalité originelle où la photographie pourrait extraire un fragment. $\mathrm{Au}$ contraire, il faut penser la restructuration que l'album opère comme la reconstruction d'une " unité ultérieure $»^{22}$. Il s'agit de penser cette unité non comme l'effet d'un ordre prédéterminé et nécessaire. On parle d'une unité qui est déjà déterminée par les multiples intentions de restructuration, avec l'aspect créatif d'un tel ordre. 

(Album de vues. Expédition à la rivière Noire. Avril à juin 1879) de Antonio Pozzo et de l'« Album de Encina, Moreno y cía 1883 » c'est parce qu'ils montrent d'une manière très claire une prise de pouvoir archivée à travers l'appareil photographique. Santa Lucía au Chili, il s'agit dans les deux cas de la conformation d'une idée de nation à partir de l'idéal moderne de progrès et de civilisation. Tel projet de nation exclurait tout autre mode d'existence qui ne s'ajusterait pas à de tels effets.

71 Si on a déjà affirmé que la modernité est structurée par une temporalité qu'on peut appeler "photographique", tout élément qui se distingue de cette temporalité moderne sera traité par une logique de caractère exclusive. Le caractère qui restera endehors sera distingué par sa temporalité « autre ». Cette temporalité autre est celle des Indiens du Sud de l'Argentine, qui à l'époque ont encore en possession des territoires. 
Les albums de Pozzo et d'Encina et Moreno font l'archivage de l'exclusion de cette autre temporalité par le gouvernement libéral de la nation argentine.

Le gouvernement d'Argentine, conscient de cette frontière à « l'intérieur » de sa propre nation, prend la décision d'en finir avec elle par le moyen de son appropriation. La prise de territoires s'est alors présentée comme un conflit entre temporalités différentes que l'appareil photographique a archivées à travers l'album, structure qui lui a donné un ordre en accord au projet moderne de la nation argentine.

Chez Schmitt, tout nomos en tant que prise de territoires est légitimé par la considération d'un territoire comme libre. Tout territoire libre, est un territoire horsnorme puisqu'il n'est pas en possession d'un nomos fondateur qui lui donne son ordre propre. On peut dire qu'un espace libre est un espace sans ordre, sans normativité, sans nomos en définitive.

Quand on examine l'album de l'expédition de la rivière Noire du photographe Pozzo (Fig. 5) ou l'album de Encina et Moreno (Fig. 6), on constate que les vues se projettent sur des territoires libres car ils se présentent comme « vides ». Dans ce sens, Alimonda et Ferguson signalent :

Significativement, ce qui est finalement souligné dans toutes les photographies est un trait précis de la perception de l'espace : le "vide » (des horizons très lointains, des terres sans limites, des grands espaces, etc. $)^{25}$

On peut dire qu'en Amérique latine il y a eu une première prise de terre pendant la conquête, qui a instauré un premier nomos sur des terres considérées comme libres. Dans un deuxième temps, il y a eu une autre prise de terre qui a eu pour conséquence un nouveau marquage du territoire, cette fois produit de l'indépendance et de l'idée de nation. Tous les deux ont procédé à la prise des territoires considérés comme vides, tandis qu'en réalité ils appartiennent aux Indiens et aux peuples autochtones. La normativité propre au monde indien n'est pas valide, car il n'existe pas d'espace commun de communication qui valide les Indiens comme intervenants légitimes, leur temporalité « autre » qui est en dehors de la civilisation va les exclure.

Le monde civilisé européen est la seule instance de légitimation possible. On peut dire que les nations en tant que telles adhèrent au projet moderne qui vient d'Europe, participent elles aussi à une temporalité qui valide leur droit à la prise de terre. Le différend temporel existant entre le conquérant européen et les Indiens d'Amérique latine va se retrouver entre les nations nouvelles et les peuples indiens.

Les albums déjà cités montrent le parcours de l'homme moderne, représentant de la nation civilisée, allant vers un territoire vide, inexploré, sans signes de civilisation. Le photographe Pozzo montre clairement comment les troupes avancent dans des territoires vides, sans résistance. Cette résistance n'a pas été photographiée, les troupes parties avant ont fait le travail d'extermination. Ce travail que Pozzo ne peut photographier remonte à l'époque de la conquête espagnole. Benjamin dit :

L'histoire coloniale des peuples européens commence par l'événement révoltant de

la conquista de l'Amérique qui transforme le monde nouvellement conquis en une salle de tortures. ${ }^{26}$

Celle salle de torture se répète, mais cette fois c'est la nation qui va faire le travail. Le photographe Pozzo a accompagné le général Roca, mais ils ont été les derniers arrivés. On peut dire qu'ils sont arrivés au moment précis où Pozzo n'a pu finalement que prendre des vues du « vide». 
79 Les terres considérées comme vides seront de nouveau le fondement de la nouvelle prise de territoires indiens, cette fois au nom de la nation argentine. Bien que les terres soient occupées par les communautés autochtones, la nation argentine ne reconnaît pas comme légitime une telle occupation. La prise de terre se présente donc comme marquage du territoire pour l'établissement d'un nomos légitime.

Mais pourquoi le peuple indien n'est-il pas considéré comme légitime? On peut dire que sa temporalité se situe hors de la modernité civilisatrice que la nation argentine promulgue. Le général Roca, chef de l'expédition signale :

Notre propre dignité en tant que peuples nous oblige à soumettre le plus tôt possible, par la raison ou par la force, une poignée de sauvages qui détruisent notre principale richesse et nous empêchent d'occuper définitivement au nom de la loi et du progrès et de notre sécurité les territoires les plus riches et fertiles de la république. ${ }^{27}$

81 Le progrès, la loi et la raison conforment le projet moderne de l'Argentine. Le monde des peuples indiens est hors-norme, il ne correspond pas au monde civilisé représenté par l'appareil photographique; leur caractère d'êtres "appropriables" sera une conséquence de ce différend temporel. Leur temporalité était différente de celle que l'idée de nation veut instaurer.

82 Il s'agit d'« objets pré-photographiques », de paysages vides, que la peinture (en tant que surface qui inscrit quelque chose en accord avec le modèle civilisé occidental) n'a pas représentés. Il n'existe donc pas de tradition connue ancrée dans ces espaces. Son apparition est due à l'appareil photographique, et son apparition s'enregistre comme un vide inoccupé parce que son occupation antérieure n'est pas valide, elle est préphotographique et pré-picturale à la fois.

83 Avant la photographie, ces espaces vides n'existaient pas pour la vision européocentrique, modèle qui, à partir de la constitution des nations en Amérique latine, continuera d'exister. Toute la temporalité moderne reçue par les nations latinoaméricaines provient de l'Europe. Le concept de vide vient de l'Europe et pourtant les nations latino-américaines continueront à regarder ces espaces de la même façon jusqu'au moment où l'appareil photographique prendra ses prises de vue et installera de façon définitive un marquage du territoire.

Si, comme le dit Ronald Kay, la photographie s'installe avant la peinture en Amérique latine, ces espaces vides en sont la preuve car ils sont "non-peints ", c'est l'appareil photographique qui les rend visibles. On ne peut pas situer une tradition occidentale dans ces espaces, ils n'ont pas été inscrits sur une surface comme la peinture, leur apparition est déterminée par la photographie, leur existence antérieure appartenait au monde du sacré. Quand l'appareil photographique prend finalement des prises de vues de ce vide il fait accéder cet espace au monde global. Kay va décrire ces espaces pré-photographiques :

Les vues photographiques de l'intérieur d'un désert, les instantanées panoramiques de la jungle ou les bouts de l'antarctique, ne sont pas un reflet de leur propre appartenance, ils impliquent plutôt d'abruptes irruptions dans le continent inconnu, des envahissements et des violations visuels d'un espace tramé par des esprits différents, aborigènes. ${ }^{28}$

Si l'appareil photographique va participer activement à la prise de territoires, c'est parce que toute prise de territoires a comme conséquence un accroissement de l'espace et parce que tout accroissement de l'espace va de pair avec un accroissement du visible. 

dit Benjamin, le chirurgien, le cameraman et le photographe montrent de quelle façon on peut pénétrer dans le tissu le plus profond de l'autre, fait manifeste de son appropriation.

\section{Figures}


Figure 1. Vicuña Mackenna, Album del Santa Lucía. Colección de las principales vistas, monumentos, jardines, estatuas i obras de este paseo. Santiago de Chile, Imprenta de la librería del Mercurio, 1874.

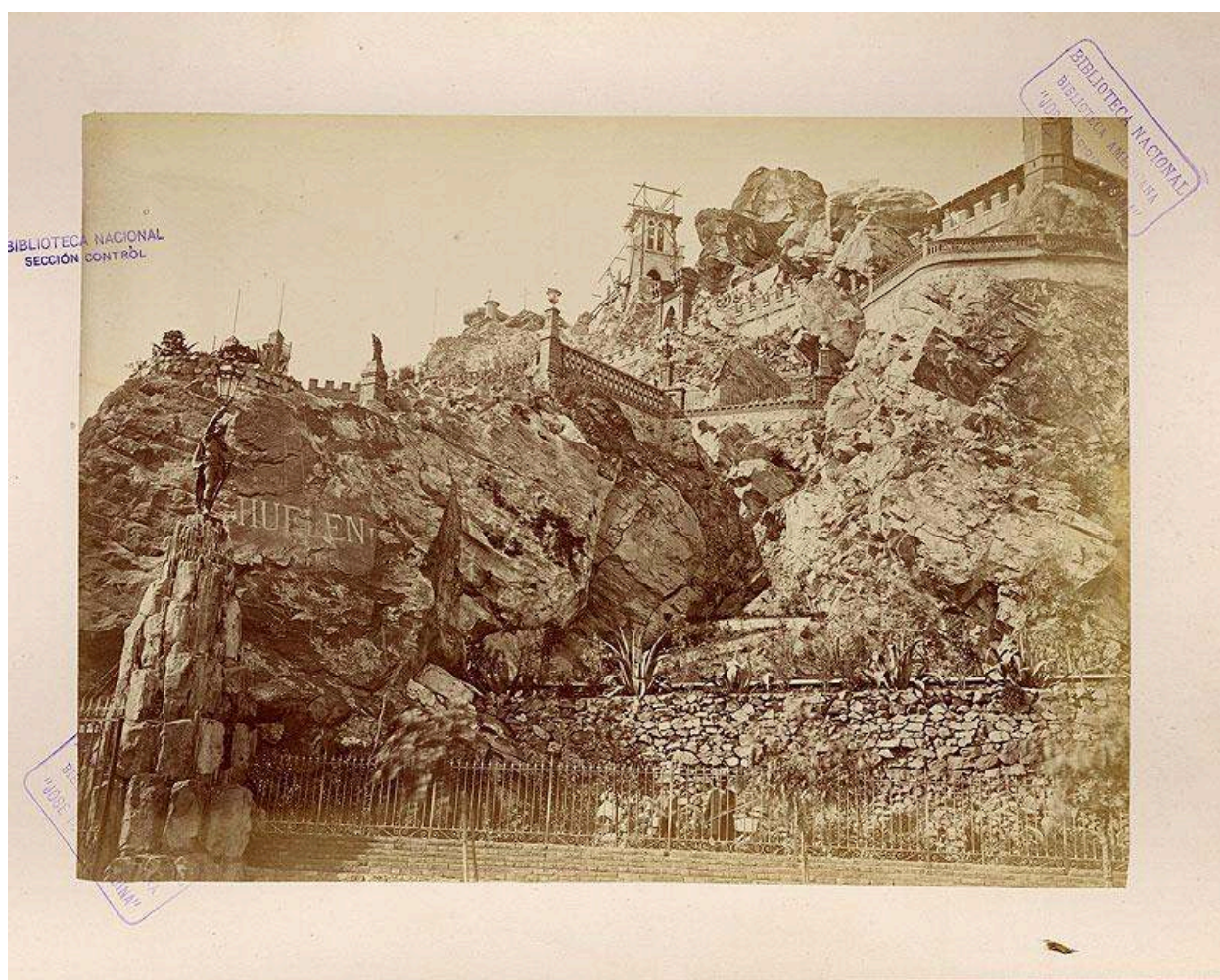

Figure 2. Vicuña Mackenna, Album del Santa Lucía. Colección de las principales vistas, monumentos, jardines, estatuas i obras de este paseo. Santiago de Chile, Imprenta de la librería del Mercurio, 1874.

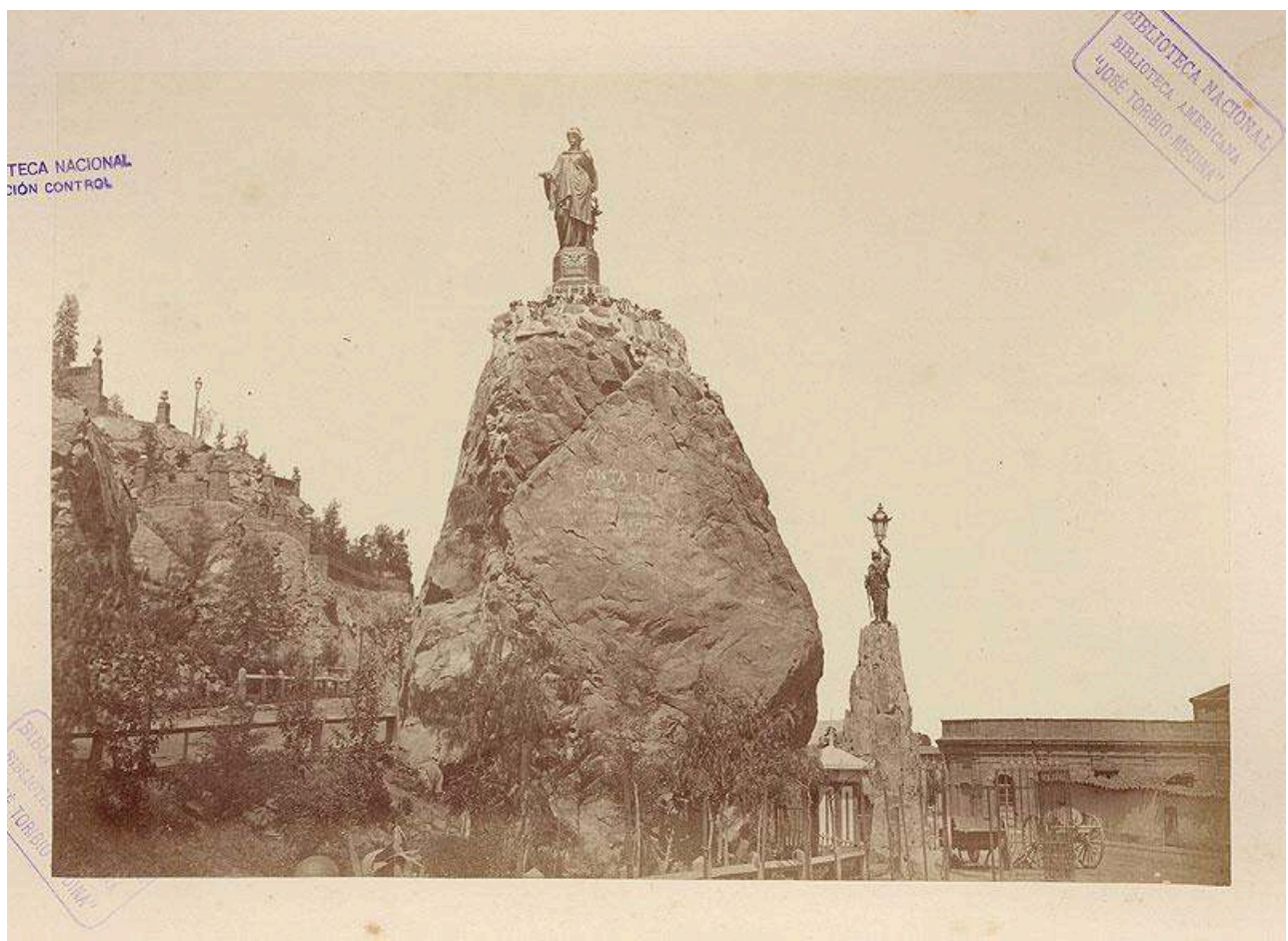


Figure 3. Musée historique-indigène. Album del Santa Lucía. Colección de las principales vistas, monumentos, jardines, estatuas i obras de este paseo. Santiago de Chile, Imprenta de la librería del Mercurio, 1874.

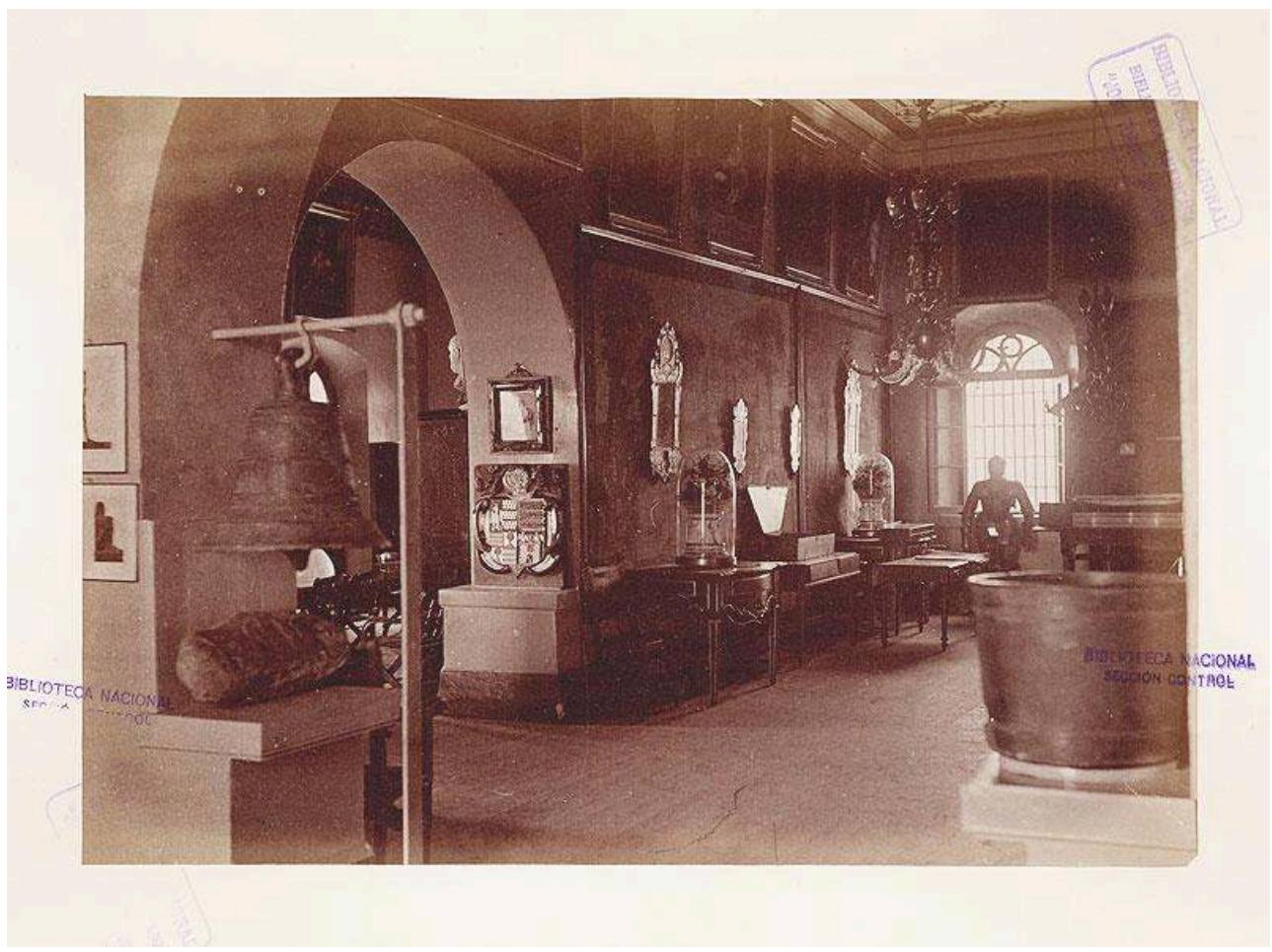

Figure 4. Couverture. Album del Santa Lucía. Colección de las principales vistas, monumentos, jardines, estatuas i obras de este paseo. Santiago de Chile, Imprenta de la librería del Mercurio, 1874.

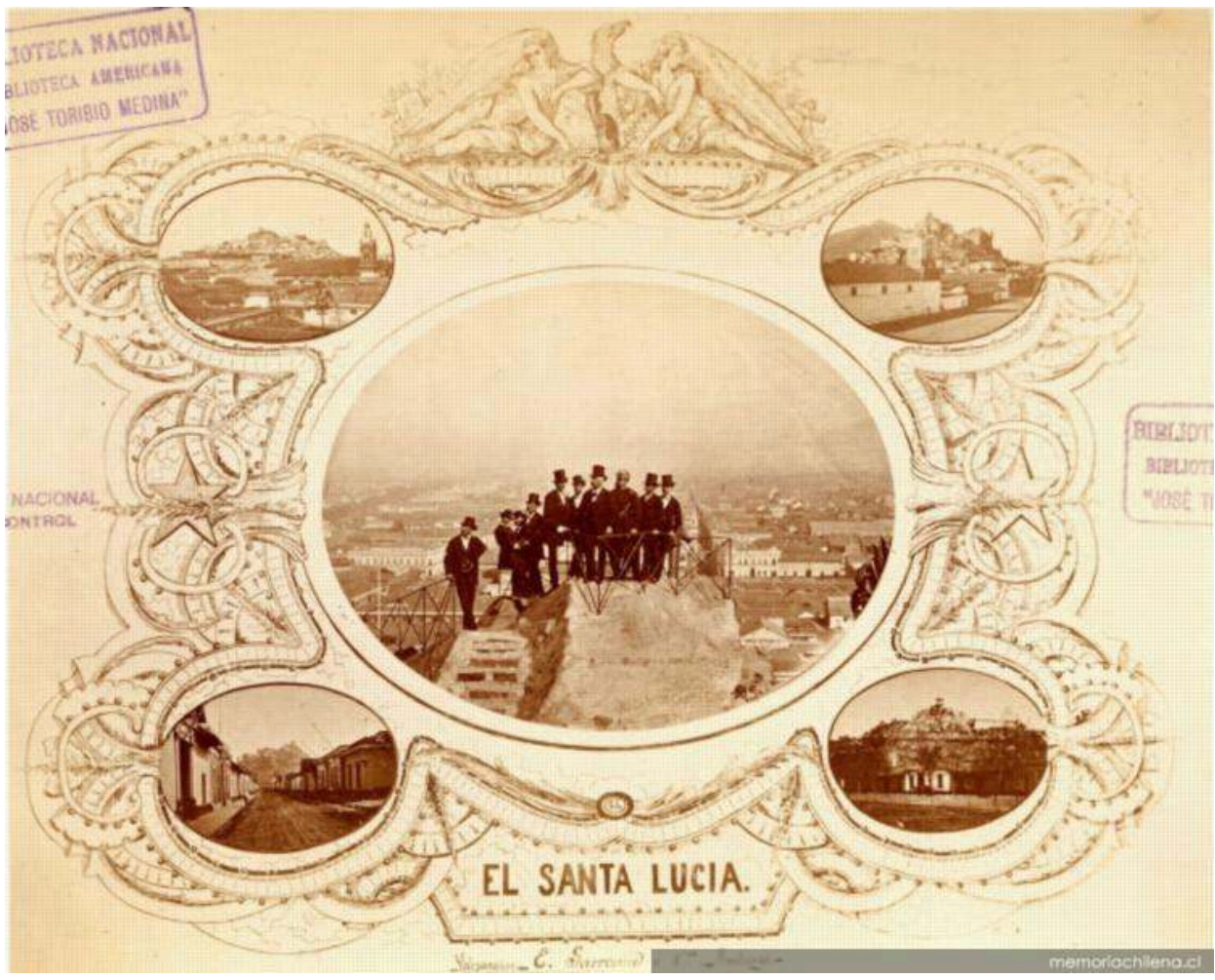


Figure 5. «El ejército marchando en columna » Album expedición al río Negro, Abril a Julio 1879

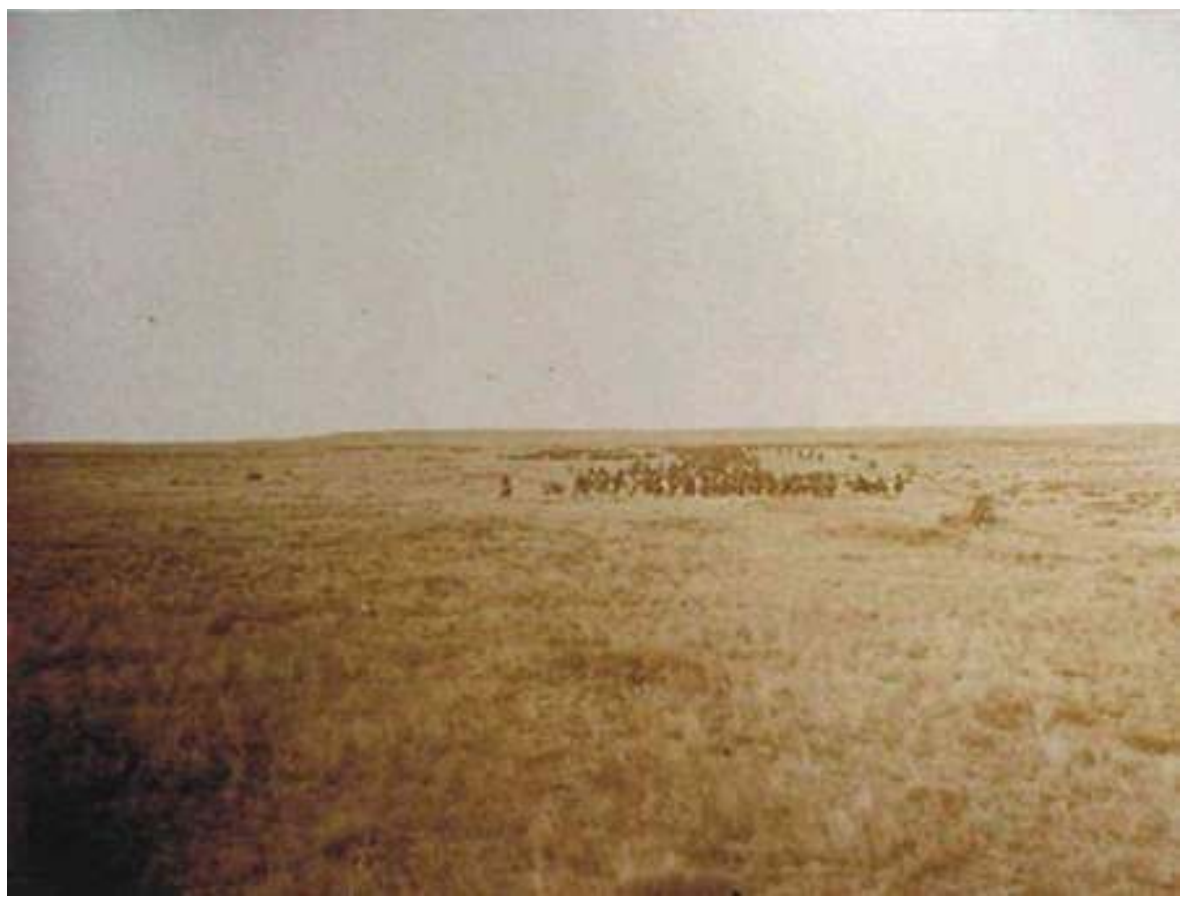

Figure 6. Caya Pelmahuida. Album Encina y Moreno, 1883

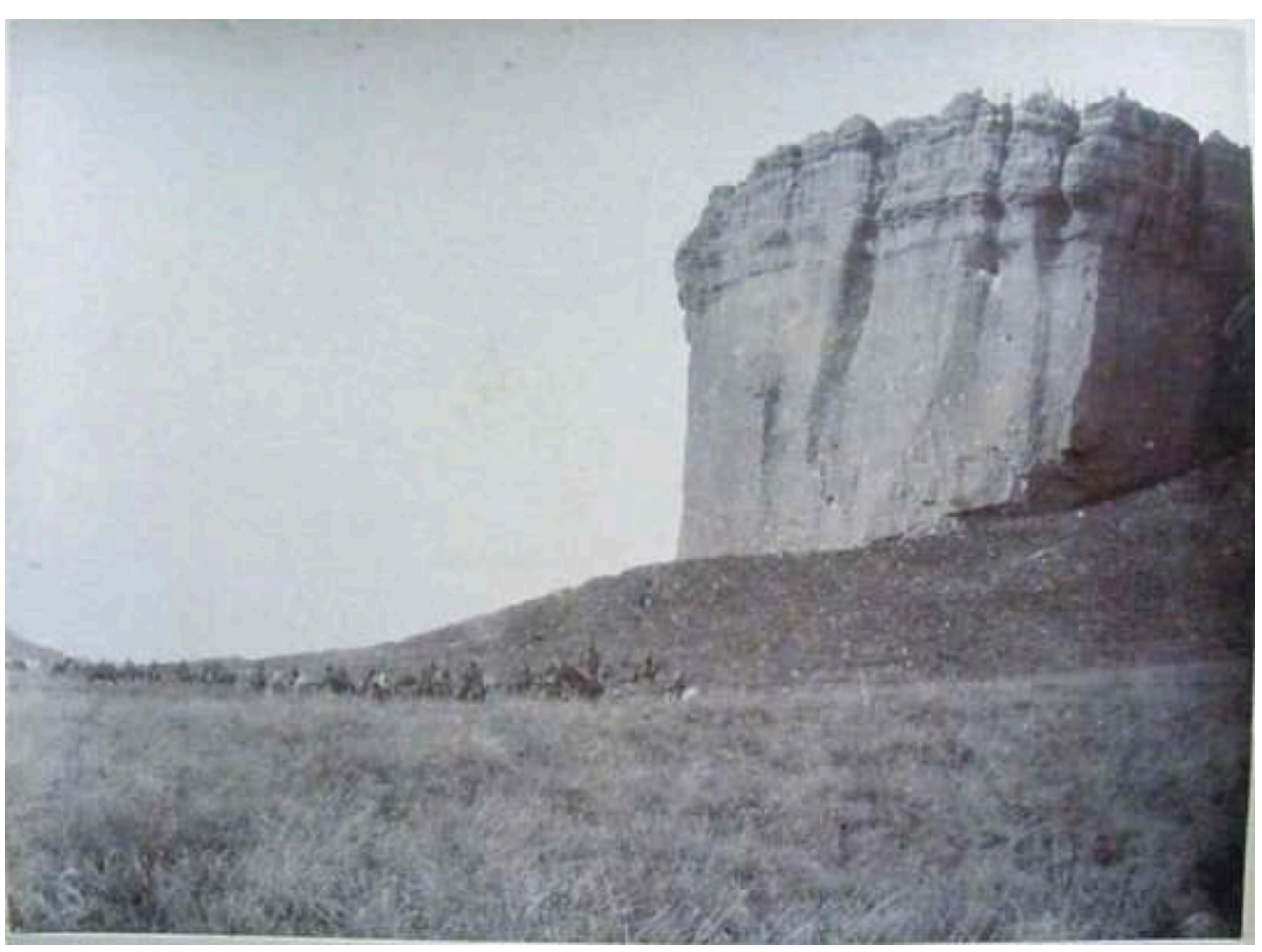




\section{BIBLIOGRAPHIE}

Alimonda Héctor et Ferguson Juan, "La producción del desierto (las imágenes de la campaña del ejército argentino contra los indios - 1879)”, Revista chilena de antropología visual, n 4 , Santiago, 2004.

Barthes Roland, La chambre claire, Paris, Cahiers du cinéma, Gallimard Seuil, 2008.

Benjamin Walter, « compte rendu de Marcel Brion, Bartholomé de las Casas, “Père des Indiens”, Plon, Paris, 1928, 309 pages » in Romantisme et critique de la civilisation, Paris, 2010.

Déotte Jean-Louis, Le musée, l'origine de l'esthétique, Paris, L'Harmattan, 1993.

Déotte Jean-Louis, L'époque des appareils, Paris, Lignes, 2004.

Derrida Jacques, Mal d'archive, Paris, Galilée, 1995.

Encina y Moreno, Album Encina y Moreno, 1883, album en ligne : http://www.museoroca.gov.ar/ index.php?option=com_content\&view=article\&id=69\&Itemid=158

Kay Ronald, Del espacio de acá. Seňales para una mirada latinoamericana, Santiago de Chile, Ediciones Metales Pesados, 2005.

Malraux André, Le musée imaginaire, Paris, Gallimard, 2008.

Meyer Alfred Gotthold, Construire en fer. Histoire et esthétique, Paris, Folio, 2005.

Pozzo Antonio, Album expedición al río Negro, Abril a Julio 1879, album en ligne : http:// www.museoroca.gov.ar/index.php?option=com_phocagallery\&view=category\&id=1\&Itemid=178 Roca Julio, “carta de Julio Roca al presidente Avellaneda, Carhué, Abril 21 de 1879” in Julio Argentino Roca. Iconografía militar, Buenos Aires, Museo Roca, Instituto de investigaciones históricas, 2006.

Rouillé André, La photographie, Paris, Gallimard, 2005.

Schmitt Carl, Le nomos de la terre, Paris, PUF, 2001.

Vicuña Mackenna Benjamin, Album del Santa Lucía. Colección de las principales vistas, monumentos, jardines, estatuas i obras de este paseo. Santiago de Chile, Imprenta de la librería del Mercurio, 1874. Album en ligne : http://www.memoriachilena.cl//temas/documento_detalle.asp ?id =MC0006534

\section{NOTES}

1. Kay Ronald, Del espacio de acá: Seňales para una mirada latinoamericana, Santiago de Chile Ediciones Metales Pesados, 2005. Traduction de Natalia Calderon.

2. Ibid., p. 29.

3. Schmitt Carl, Le nomos de la terre, Paris, PUF, 2001.

4. Ibid., p. 70.

5. Derrida Jacques, Mal d'archive, Galilée, Paris, 1995.

6. Barthes Roland, La chambre claire, Paris, Cahiers du cinéma, Gallimard Seuil, 2008, p. 136.

7. Derrida Jacques, op. cit., p. 13.

8. Déotte Jean-Louis, L'époque des appareils, Paris, Lignes, 2004, p. 51.

9. Plusieurs sont les critiques à l'idée ontologique de la photographie. Pour une intéressante critique sur Ronald Barthes, cf. Rouillé André, La photographie, Paris, Gallimard, 2005. 
10. Vicuña Mackenna Benjamin, Album del Santa Lucía. Colección de las principales vistas, monumentos, jardines, estatuas i obras de este paseo, Santiago de Chile, Imprenta de la librería del Mercurio, 1874.

11. Pozzo Antonio, Album expedición al río Negro, Abril a Julio 1879.

12. Encina y Moreno, Album Encina y Moreno, 1883.

13. Schmitt, op. cit., p. 83.

14. L'œuvre de Pedro Lira, La fondation de Santiago, est la représentation de cet événement de la prise de terre par Pedro de Valdivia.

15. Vicuña Mackenna Benjamin, op. cit., p. V. Traduction de Natalia Calderon.

16. Ibid, p. VI-VII.

17. Meyer Alfred Gotthold Meyer, Alfred Gotthold, Construire en fer. Histoire et esthétique, Paris, Folio, 2005, p. 15.

18. Malraux André, Le musée imaginaire, Paris, Gallimard, 2008, p. 257.

19. Déotte Jean-Louis, Le musée, l'origine de l'esthétique, Paris, L'Harmattan, 1993, p. 87.

20. Vicuña Mackenna, op. cit., p. 3.

21. Rouillé André, op. cit., p. 124.

22. Ibid., p. 130.

23. Derrida Jacques, op. cit., p. 14.

24. On peut déjà retrouver telle configuration temporelle dans le baroque colonial. Sans doute, il s'agit de l'idée d'un monde global que la découverte d'un nouveau continent peut avoir apporté. Mais tel rassemblement spatio-temporel obéit à un ordre transcendantal qui le rend possible, car il s'agit dans ce cas-là d'omniscience divine. On peut dire que l'appareil photographique rend humaine cette omniscience comme conquête de l'ubiquité.

25. Alimonda Héctor et Ferguson Juan, "La producción del desierto (las imágenes de la campaña del ejército argentino contra los indios - 1879)" in Revista chilena de antropología visual, $\mathrm{n}^{\circ} 4$, Santiago, 2004, p. 8. Traduction de Natalia Calderon.

26. Benjamin Walter, « compte rendu de Marcel Brion, Bartholomé de las Casas, "Père des Indiens", Paris, Plon, 1928, 309 pages » in Romantisme et critique de la civilisation, 2010, p. 129.

27. Roca Julio, "carta de Julio Roca al presidente Avellaneda, Carhué, Abril 21 de 1879" in Julio Argentino Roca. Iconografía militar, Buenos Aires, Museo Roca, Instituto de investigaciones históricas, 2006, p. 32, traduction de Natalia Calderon.

28. Kay Ronald, op. cit., p. 28, traduction de Natalia Calderon.

\section{RÉSUMÉS}

L'appropriation de l'appareil photographique par les gouvernements nationaux en Amérique latine va provoquer une coupure temporelle et politique. Cette coupure déterminera le visible et l'invisible dans les différents imaginaires nationaux naissants.

\section{INDEX}

Mots-clés : album, appareil photographique, archive, études post-coloniales, C. Schmitt, prise de vue 
AUTEUR

NATALIA CALDERÓN

Doctorante en philosophie, université Paris 8, laboratoire d'études et de recherches sur le logiques contemporaines de la philosophie, martinez.talanatalia@gmail.com 